\title{
The genetic basis for inactivation of Wnt pathway in human osteosarcoma
}

\author{
Xiaoling Du ${ }^{1,2,4+}$, Jilong Yang ${ }^{2,3^{*}+}$, Da Yang ${ }^{3+}$, Wei Tian ${ }^{2}$ and Ze Zhu ${ }^{4 *}$
}

\begin{abstract}
Background: Osteosarcoma is a highly genetically unstable tumor with poor prognosis. We performed microarray-based comparative genomic hybridization (aCGH), transcriptome sequencing (RNA-seq), and pathway analysis to gain a systemic view of the pathway alterations of osteosarcoma.

Methods: aCGH experiments were carried out on 10 fresh osteosarcoma samples. The output data (Gene Expression Omnibus Series accession number GSE19180) were pooled with published aCGH raw data (GSE9654) to determine recurrent copy number changes. These were analyzed using Kyoto Encyclopedia of Genes and Genomes (KEGG) pathway analysis to identify altered pathways in osteosarcoma. Transcriptome sequencing of six osteosarcomas was performed to detect the expression profile of Wnt signaling pathway genes. Protein expression of WNT1, $\beta$-catenin, c-myc, and cyclin D1 in the Wnt pathway was detected by immunohistochemistry (IHC) in an independent group of 46 osteosarcoma samples.
\end{abstract}

Results: KEGG pathway analysis identified frequent deletions of Wnt and other Wnt signaling pathway genes. At the mRNA level, transcriptome sequencing found reduced levels of mRNA expression of Wnt signaling pathway transcripts. While WNT1 protein expression was detected by IHC in 69.6\% (32/46) of the osteosarcomas, no $\beta$-catenin protein was detected in the nucleus. $\beta$-catenin protein expression was, however, detected in the membrane and cytoplasm of $69.6 \%$ (32/46) of the osteosarcomas. c-myc protein expression was detected in only 47.8\% (22/46) and cyclin D1 protein expression in 52.2\% (24/46) of osteosarcoma samples. Kaplan-Meier survival analysis showed that WNT1-negative patients had a trend towards longer disease free survival than WNT1-positive patients. Interestingly, in WNT1-negative patients, those who were also cyclin D1-negative had significantly longer disease free survival than cyclin D1-positive patients. However, there was no significant association between any of the investigated proteins and overall survival of human osteosarcoma patients.

Conclusions: Frequent deletions of Wnt and other Wnt signaling pathway genes suggest that the Wnt signaling pathway is genetically inactivated in human osteosarcoma.

Keywords: Osteosarcoma, Wnt signal pathway, Genetic aberration, Microarray-based comparative genomic hybridization

\section{Background}

Osteosarcoma is a malignant bone tumor, often associated with copy number alterations, that most commonly arises in the metaphyseal ends of long bones [1-3]. The survival of patients with osteosarcoma has not improved significantly in recent years and the prognosis of patients

\footnotetext{
*Correspondence: yangjilong@tjmuch.com; zhuze_2006@126.com

${ }^{+}$Equal contributors

${ }^{2}$ Department of Bone and Soft Tissue Tumors, Tianjin Medical University Cancer Institute \& Hospital, National Clinical Research Center for Cancer, Tianjin 30060, China

${ }^{4}$ Department of Medical Microbiology, Tianjin Medical University, Tianjin 300060, China

Full list of author information is available at the end of the article
}

with metastatic osteosarcoma is especially poor [1]. Identification of prognosis markers and key genetic and molecular events for osteosarcoma is critical for development of effective therapeutics [2,3].

Fortunately, the discovery of signal transduction pathways and their importance in a variety of cancers has led to the development of many new targeted agents. With regard to osteosarcoma, preclinical investigations targeting the rapamycin (mTOR) pathway, as well as the VEGF pathway showed promising results [2,4]. The Wnt pathway is clearly important in many forms of human cancer, particularly in epithelial cancer types where gain- 
or loss-of-function events appear to contribute to both inherited cancer risk and somatic carcinogenesis [5]. In human osteosarcoma, most previous studies have suggested that active Wnt signaling contributes to osteosarcoma development, evidenced by cytoplasmic and/or membranous $\beta$-catenin staining or detection of Wnt pathway components [6,7]. However, Cai and colleagues recently reported that the Wnt pathway is inactivated in osteosarcomas [8]. These contradictory findings provoke debate and stimulate further research into the role of Wnt signaling in osteosarcoma [5].

In this study, we sought to gain a comprehensive understanding of the key driving pathways for osteosarcoma by performing pathway analysis of recurring gene copy number aberrations using array comparative genomic hybridization $(\mathrm{aCGH})$ analysis. Transcriptome sequencing (RNA-seq) and immunohistochemistry (IHC) were used to explore the expression level of key signaling pathways affected by these genetic aberrations. The most surprising and intriguing finding was the deletion of Wnt pathway genes, suggesting the genetic inactivation of the Wnt signaling pathway, in human osteosarcoma.

\section{Methods}

\section{Osteosarcoma tissues and clinical information}

Ten frozen osteosarcoma biopsy samples, all with at least $90 \%$ tumor content, were obtained from the Tissue Bank of the Tianjin Medical University Cancer Institute \& Hospital (TMUCIH) for aCGH and RNA-Seq analysis as described below. Tumor samples were snap frozen in liquid nitrogen. In addition, an independent group of 46 primary osteosarcoma cases with formalin-fixed and paraffin-embedded (FFPE) tissues and clinicopathologic data were collected. All of the tissues and information collection took place at Tianjin Medical University Cancer Institute \& Hospital (TMUCIH) with Institutional Review Board (IRB) approved protocols and the patients' consent. The clinical and pathological parameters included age, gender, locations, Enneking staging, and follow-up data (Table 1). All neoadjuvant and adjuvant chemotherapy had been administered according to the Rosen T10 regimen [9,10]. Disease-free and overall survival time ranged from 0 to 94 months, with medians of 9 and 13 months, respectively.

\section{Array comparative genomic hybridization and pathway enrichment analysis}

The aCGH data analysis was performed as previously described [2,3]. aCGH data in this publication have been deposited in NCBI's Gene Expression Omnibus (GEO) and are accessible through GEO Series accession number GSE19180. In addition, we obtained the raw aCGH data of another 10 osteosarcoma biopsy samples from the GEO database (GSE9654) [11] and pooled the two datasets for analysis. Briefly, the median-normalized log2 ratio data were first subjected to a circular binary segmentation (CBS) algorithm to reduce the effect of noise [12]. Then, the CGHcall algorithm was used to call segments of DNA sequences as amplified or deleted in each sample [13]. A permutation analysis was further applied to call recurrent copy number aberration in osteosarcoma [2]. As a result of this procedure, each target was given a label of "normal", "deletion" or "amplification" as previously described $[2,3,13]$. A bacterial artificial chromosome (BAC) clone-based CGH array dataset from 36 cases of osteosarcoma was also analyzed to confirm the overall recurrent gene copy alteration patterns $[2,3,14]$.

Pathway enrichment analysis was performed separately on the recurrent amplified and deleted gene lists from gene sets GSE19180 and GSE9654 as previously reported $[2,3]$. Here, we used the gene annotations data in the Kyoto Encyclopedia of Genes and Genomes (KEGG). Pathways with more annotations in our gene list than expected by random (hypergeometric model, $\mathrm{P}<0.05$ ) were considered to be significantly enriched with amplified or deleted genes $[2,3]$.

\section{RNA-seq analysis}

Frozen tumors were crushed, then total RNA was isolated using TRIzol reagent (Invitrogen, Grand Island, NY). RNA was quantified by Qubit (Invitrogen, Grand Island, NY) and Nanodrop ND1000 (ThermoFisher Scientific, Waltham, MA) before quality assessment with the Agilent 2100 Bioanalyzer. Six of the 10 samples with high quality RNA were used for RNA library construction, followed by emulsion PCR and whole transcriptome $90 \mathrm{bp}$ paired-end sequencing on Illumina $\mathrm{HiSeq}^{\text {Tix }} 2000$ instruments at the Beijing Genomics Institute (BGI, Shenzhen, China). Each sequencing run produced approximately 50 million paired end reads.

Whole transcriptome sequencing reads were aligned against the GRCh37 human reference genome using Tophat version 2.0.4 [15]. The number of overlapping reads was calculated for all exons and then for all genes annotated in Ensembl 67. Gene expression values were normalized across samples using median-of-ratios normalization [16]. Briefly, an expression ratio between two samples for every gene (or exon) with $>500$ reads is calculated, and the median of those ratios is determined. All gene expression values are then multiplied by the median-of-ratios.

Immunohistochemical analysis of WNT1, $\beta$-catenin, c-myc, and cyclin D1 expression

The 46 FFPE tissues were sectioned at $4 \mu \mathrm{m}$ and mounted onto charged glass slides (ProbeOn Plus, Fisher Scientific, Pittsburgh, PA, USA) for immunohistochemical staining as described previously [2]. Briefly, tissues were deparaffinized 
Table 1 The clinical and pathological features in $\mathbf{4 6}$ human conventional osteosarcomas

\begin{tabular}{|c|c|c|c|c|c|c|c|c|c|c|}
\hline & & \multirow[t]{3}{*}{$\mathrm{N}$} & \multicolumn{4}{|c|}{ Disease free survival } & \multicolumn{4}{|c|}{ Overall survival } \\
\hline & & & \multirow[t]{2}{*}{$\bar{P}$} & \multirow[t]{2}{*}{ HR } & \multicolumn{2}{|c|}{$95.0 \% \mathrm{Cl}$} & \multirow[t]{2}{*}{$\bar{P}$} & \multirow[t]{2}{*}{ HR } & \multicolumn{2}{|c|}{$95.0 \% \mathrm{Cl}$} \\
\hline & & & & & $\mathrm{L}$ & $U$ & & & $\mathrm{~L}$ & $U$ \\
\hline \multirow[t]{2}{*}{ Sex } & Male & 29 & 0.86 & & & & $0.07^{*}$ & & & \\
\hline & Female & 29 & 0.86 & 1.11 & 0.36 & 3.44 & 0.08 & 3.49 & 0.86 & 14.28 \\
\hline \multirow[t]{5}{*}{ Age group } & $<=15 \mathrm{y}$ & 14 & 0.76 & & & & 0.79 & & & \\
\hline & $15-20 y$ & 23 & 0.94 & $1.10 \mathrm{E}+04$ & 0 & $1.40 \mathrm{E}+116$ & 0.5 & 1.82 & 0.32 & 10.42 \\
\hline & $21-30 y$ & 11 & 0.94 & $3.70 E+04$ & 0 & $4.40 E+116$ & 0.72 & 0.73 & 0.13 & 4.05 \\
\hline & $31-40$ y & 1 & 0.93 & $5.30 E+04$ & 0 & $6.40 E+116$ & 0.97 & 0 & 0 & $1.90 E+282$ \\
\hline & $>40 y$ & 9 & 1 & 1.02 & 0 & $3.90 E+225$ & 0.99 & 0 & 0 & \\
\hline \multirow[t]{2}{*}{ Tumor location } & limbs & 51 & 0.28 & & & & 0.97 & & & \\
\hline & Others & 7 & 0.48 & 23.34 & 0.004 & $1.60 E+05$ & 0.97 & 0.97 & 0.12 & 7.72 \\
\hline \multirow[t]{4}{*}{ Enneking stage } & । & 4 & 0.96 & & & & 0.43 & & & \\
\hline & $\| A$ & 19 & 0.78 & 0.71 & 0.06 & 7.88 & 0.17 & 0.1 & 0.004 & 2.74 \\
\hline & $\| B$ & 2 & 0.631 & 0.59 & 0.07 & 5.01 & 0.1 & 0.09 & 0.01 & 1.61 \\
\hline & III & 1 & 0.897 & 0.83 & 0.05 & 13.39 & 0.99 & 0 & 0 & \\
\hline \multirow[t]{2}{*}{ Neoadjuvant chemotherapy } & No & 10 & 0.51 & & & & $1.5 \mathrm{E}-4^{*}$ & & & \\
\hline & Yes & 30 & 0.51 & 1.51 & 0.44 & 5.15 & 0.003 & 11.85 & 2.36 & 59.45 \\
\hline \multirow[t]{2}{*}{ Adjuvant chemotherapy } & No & 3 & 0.21 & & & & 0.41 & & & \\
\hline & Yes & 19 & 0.43 & 0.04 & 0 & 129.34 & 0.61 & 0.04 & 0 & $1.20 E+04$ \\
\hline \multirow[t]{2}{*}{ Recurrence } & No & 50 & & & & & 0.49 & & & \\
\hline & Yes & 8 & & & & & 0.49 & 2.05 & 0.26 & 16.26 \\
\hline \multirow[t]{2}{*}{ Metastasis } & No & 38 & & & & & $0.045^{*}$ & & & \\
\hline & Yes & 11 & & & & & 0.436 & 0.01 & 0 & $2.40 E+03$ \\
\hline
\end{tabular}

*Significantly different; L: lower; U: upper.

with xylene and ethanol, endogenous peroxidase activity was blocked with $0.3 \% \mathrm{H}_{2} \mathrm{O}_{2}$ (Fisher Scientific, Fair Lawn, NJ, USA). Tissues were blocked for $30 \mathrm{~min}$ with normal serum (Vector Laboratories, Burlingame, CA, USA) and then incubated overnight at $4^{\circ} \mathrm{C}$ with appropriate antibodies. Antibodies for WNT1, $\beta$-catenin, c-myc, and cyclin D1 (Abcam, Cambridge, UK) were used at a dilution of 1:200, 1:100, 1:100, and 1:100, respectively. The same concentrations of non-immune rabbit serum were used as negative controls. Signal was detected using biotinylated anti-rabbit antibodies, followed by avidin, biotinylated enzyme and colorimetric detection using 3,3'-diaminobenzidine tetrahydrachloride (DAB) (DAKO Corporation, Carpinteria, CA, USA). Samples were then counterstained with Mayer's hematoxylin (Polyscientific, Bay Shore, NY, USA).

Two pathologists, blinded to clinical information, evaluated and scored the immunohistochemical staining for WNT1, $\beta$-catenin, c-myc, and cyclin D1 in osteosarcoma tissues based on the overall intensity of membranous, cytoplasmic, and nuclear staining within the tumor cells and the percentage of cells stained [8,17-20]. WNT1 and $\beta$-catenin staining were scored as described in $[8,17]$. Specifically, intensity of staining was graded as follows: lost (score 0); weak ("+", score 1); moderate (“++", score 2); and strong ("+++", score 3). Extent of staining was evaluated as the percentage of positive cells per 100 or more cells (at least 100 cells in 10 high-power fields) in each evaluated compartment and was graded into five classes as follows: $<5 \%$ (score 0 ); $6 \%$ to $25 \%$ (score 1); $26 \%$ to $50 \%$ (score 2); $51 \%$ to $75 \%$ (score 3 ); $>75 \%$ (score 4 ). A final staining score was calculated by adding intensity score and extent score for each compartment, and the expression was categorized into two classes based on the final score: positive (final score 2-7) and negative (final score 0-1) expression. For $\beta$-catenin staining evaluation, any intensity and extent of staining found in the nucleus was considered positive. The staining of c-myc and cyclin D1 was assessed on the basis of published data: negative $(<10 \%$ of the cells), and positive ( $>10 \%$ of the cells) [18-20].

\section{Statistics}

Student's t-test, ANOVA, Chi-square, Fisher's exact test, Kaplan-Meier, and Mantel-Cox survival analysis were 
performed using SPSS software 16.0 version when necessary. A P value of less than 0.05 was considered statistically significant in multivariate analysis.

\section{Results}

\section{Several signaling pathways are genetically altered in} osteosarcoma

As presented in our previous reports, high-density genomewide aCGH profiling (GSE19180) of 10 osteosarcoma tissues identified several major regions with significant genetic alterations. This pattern of copy number alterations was strikingly similar to that from an independent aCGH dataset (GSE9654) of osteosarcoma samples obtained from Canadian patients $[2,3,11]$. It was therefore reasonable to pool the two aCGH datasets (GSE19180 and GSE 9654) for further pathway analysis. Analysis of the combined dataset led to the identification of the amplification of 2,519 genes and deletion of 1,276 genes among the 20 osteosarcoma samples (Figure 1A). We also analyzed a BAC clonebased, aCGH dataset from 36 Norwegian osteosarcoma samples (Figure 1B) [14]. The analysis of this third dataset showed similar results to the first 20 aCGH osteosarcomas, suggesting consistent genetic alterations underlying the pathogenesis of osteosarcoma.

The GSE19180 and GSE9654 datasets were measured on Agilent Human Genome CGH Microarrays, while the third aCGH dataset was BAC clone-based. Therefore the KEGG pathway enrichment analysis was only performed on dataset GSE19180 and GSE9654. In the KEGG pathway enrichment analysis, significant genetic amplifications were identified in the component genes of 20 signaling pathways including the VEGF, mTOR, CAMs, and adherens junction signaling pathways. Significant genetic deletions were identified in the component genes of 11 signaling pathways including the Wnt and Hedgehog signaling pathways (Tables 2 and 3). In our previous studies, the integrated methods of aCGH, fluorescent in situ hybridization (FISH), and IHC has led to validation of the genetic amplification of VEGF pathway genes [2].

\section{Wnt pathway genes are deleted in human osteosarcomas} In contrast to the amplification of VEGF pathway genes, we detected significant over-representation of deleted genes in 11 pathways. Among these pathways, the Wnt signaling pathway was most highly affected (Table 3). This is the first description of this genetic aberration in human osteosarcoma. As controversy exists in the field as to whether the Wnt signaling pathway is inactivated or not in osteosarcoma $[8,21]$, we further investigated copy number alterations of individual genes in the Wnt signaling pathway. In the canonical Wnt signaling pathway, the following genes were significantly deleted across the osteosarcoma dataset: WNT, FRP, Frizzled, GBP, GSK-3 $\beta, T C F /$ $L E T$, TAK1, CK1, CtBP, and $B-\operatorname{Tr} C P$ (Figure 2). Specifically, the WNT1 gene was deleted in 10 cases of the 20 human osteosarcomas with a deletion frequency of $50 \%$.

\section{Reduced transcript and protein expression of Wnt signaling pathway components suggests the Wnt signaling pathway is inactivated in human osteosarcomas} To determine whether there was an association between gene copy number and mRNA expression of Wnt pathway genes, we compared transcriptome sequencing data of six osteosarcoma samples (Additional file 1) with aCGH analysis performed on their genomic DNA (Additional file 2). We found that the deletion of certain genes was associated with low mRNA expression, such as $N L K, S O X 1, M A P K 8$, $M A P 1 B$, and FZD7 genes (Figure 3).

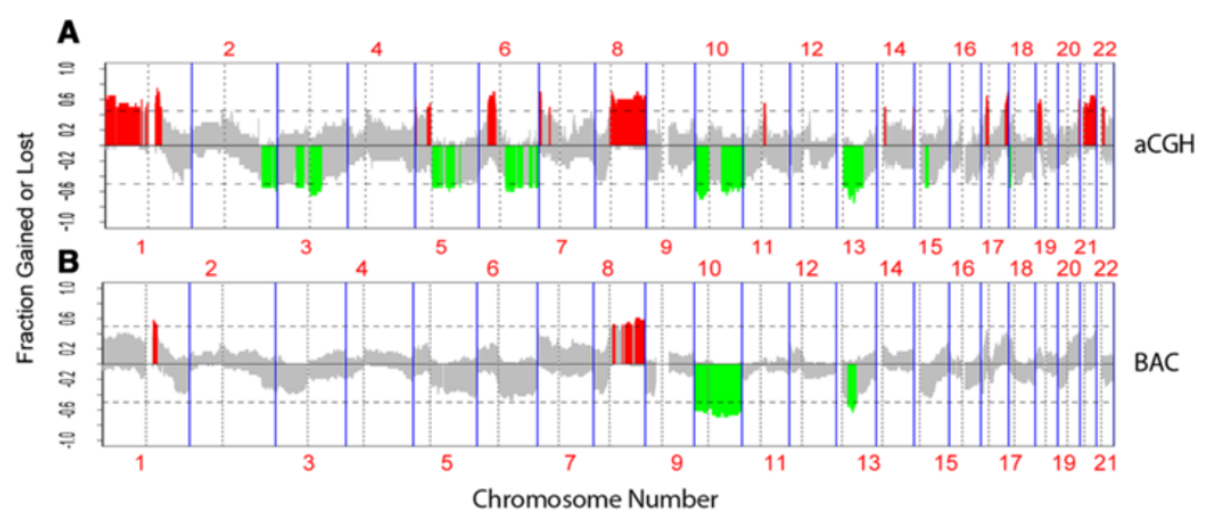

Figure 1 Genetic aberrations in human osteosarcoma samples. The x-axis numbered with 1-22 denotes chromosome numbers. The y-axis denotes log-ratio for every aCGH probe (scatter plot). The $y$-axis shows recurrence of gains (positive axis) and losses (negative axis) for each measured sequence aligned evenly in chromosomal order on the x-axis. Recurrence rates that exceed the threshold are color-coded to emphasize the locations of significantly recurrent aberrations. Red denotes significantly recurrent amplifications and green denotes significantly recurrent deletions. Gray represents nonsignificant recurrence of aberrations. A. Genetic aberrations of 20 osteosarcomas (GSE19180 and GSE9654). B. Genetic aberrations of 36 Norwegian osteosarcoma samples. 
Table 2 Genetic amplifications of key pathway genes in osteosarcoma

\begin{tabular}{|c|c|c|}
\hline KEEG pathways & P-value & Gene name cohort \\
\hline VEGF signaling pathway & 0.000611811 & $\begin{array}{l}\text { MAPK14, AKT1, PLA2G2D, PLA2G2E, NFATC4, PIK3CD, PLA2G2A, PLA2G5, MAPK1, MAPK13, PTK2, } \\
\text { RAC1, RAC3, PLA2G2F, VEGFA, CASP9, PIK3R3, SPHK1, SH2D2A, CDC42P2, CDC42 }\end{array}$ \\
\hline mTOR signaling pathway & 0.024894273 & AKT1, MTOR, RICTOR, PIK3CD, PRKAA1, PRKAA2, MAPK1, RPTOR, RPS6KA1, VEGFA, PIK3R3, ULK2 \\
\hline Tight junction & 0.044332801 & $\begin{array}{l}\text { INADL, EXOC3, CSNK2B, CLDN19, AKT1, CLDN14, CLDN17, LLGL2, LLGL1, MYH6, MYH7, F11R, ASH1L, } \\
\text { PRKCZ, CGN, JAM2, RAB3B, RAB13, ACTB, ACTG1, CLDN5, CLDN8, CRB3, TJAP1, CDC42P2, CDC42 }\end{array}$ \\
\hline $\begin{array}{l}\text { Synthesis and degradation } \\
\text { of ketone bodies }\end{array}$ & 0.019871373 & HMGCL, HMGCS1, HMGCS2, OXCT1 \\
\hline C21-Steroid hormone metabolism & 0.04221587 & CYP11B1, CYP11B2, HSD3B1, HSD3B2 \\
\hline Peptidoglycan biosynthesis & 0.017208179 & PGLYRP2, PGLYRP3, PGLYRP4 \\
\hline Ether lipid metabolism & 0.006547877 & AGPAT1, PLA2G2D, PLA2G2E, PAFAH2, ENPP2, PLA2G2A, PLA2G5, AGPAT3, PLA2G2F, PPAP2B \\
\hline Arachidonic acid metabolism & 0.02227667 & $\begin{array}{l}\text { CYP2J2, CYP4A11, GPX6, PLA2G2D, GPX5, GPX7, PLA2G2E, CYP4F3, PLA2G2A, PLA2G5, PLA2G2F, } \\
\text { CYP4F2, CBR3 }\end{array}$ \\
\hline Alpha-Linolenic acid metabolism & 0.003362937 & PLA2G2D, PLA2G2E, ACOX1, PLA2G2A, PLA2G5, PLA2G2F, FADS2 \\
\hline Nitrogen metabolism & 0.007842327 & CTH, CA14, CA1, CA2, CA3, CA6, CA8, ASRGL1 \\
\hline Glycan structures-biosynthesis 2 & 0.025972282 & $\begin{array}{l}\text { PIGK, B3GALT5, ST6GALNAC3, PIGP, PIGV, ST3GAL1, ST3GAL3, ST6GALNAC5, B4GALT3, B4GALT2, } \\
\text { B3GALT4, GPAA1, PIGM, PIGL }\end{array}$ \\
\hline $\begin{array}{l}\text { Biosynthesis of unsaturated } \\
\text { fatty acids }\end{array}$ & 0.021314211 & ACOT7, FASN, ACOT11, FADS1, ACOX1, FADS2, TECR \\
\hline $\begin{array}{l}\text { Antigen processing and } \\
\text { presentation }\end{array}$ & 0.028228218 & $\begin{array}{l}\text { CTSS, HLA-DMA, HLA-DMB, HLA-DOA, HLA-DOB, HLA-DPA1, HLA-DQA2, HLA-DRB5, HLA-F, } \\
\text { HSPA1B, HSPA1A, HSPA1L, HSP9OAB1, NFYA, NFYC, RFX5, TAP1, TAP2, CALR }\end{array}$ \\
\hline Fc epsilon RI signaling pathway & 0.000481297 & $\begin{array}{l}\text { MAPK14, AKT1, FCER1A, FCER1G, PLA2G2D, GRB2, PLA2G2E, LYN, PIK3CD, PLA2G2A, PLA2G5, } \\
\text { MAPK1, MAPK13, MAP2K3, MAP2K7, RAC1, RAC3, PLA2G2F, TNF, VAV1, PIK3R3 }\end{array}$ \\
\hline Insulin signaling pathway & 0.047775005 & $\begin{array}{l}\text { FLOT1, CRKL, AKT1, FASN, EXOC7, MTOR, GRB2, INSR, PFKL, PIK3CD, PKLR, PRKAA1, PRKAA2, } \\
\text { PRKAB2, PRKACA, PRKACB, PRKCZ, MAPK1, RPTOR, PTPRF, SHC1, SREBF1, PIK3R3, MKNK1, TRIP10 }\end{array}$ \\
\hline GnRH signaling pathway & 0.04873803 & $\begin{array}{l}\text { ADCY8, MAPK14, ADCY4, PLA2G2D, GRB2, PLA2G2E, ITPR3, JUN, PLA2G2A, PLA2G5, PRKACA, } \\
\text { PRKACB, MAPK1, MAPKT, MAPK13, MAP2K3, MAP2K7, PLA2G2F, CDC42P2, CDC42 }\end{array}$ \\
\hline Alzheimer's disease & 0.020850782 & NCSTN, BACE2, APP, APH1A, C1QA, TNF, C1QB, C1QC \\
\hline Asthma & 0.003038215 & FCER1A, FCER1G, HLA-DMA, HLA-DMB, HLA-DOA, HLA-DOB, HLA-DPA1, HLA-DQA2, HLA-DRB5, TNF \\
\hline Systemic lupus erythematosus & 2.49E-06 & $\begin{array}{l}\text { FCGR1A, FCGR2A, FCGR2B, FCGR3A, HIST1H2AB, HIST1H2AE, HIST1H2BD, H3F3B, H3F3A, HLA-DMA, } \\
\text { HLA-DMB, HLA-DOA, HLA-DOB, HLA-DPA1, HLA-DQA2, HLA-DRBS, HIST2H2AB, CIQA, TNF, C1QB, } \\
\text { CIQC, C2, C3, C6, C7, C8A, C8B, C9, HIST1H2AG, SMCHDI, HIST1H2AI, HIST1H2AK, HIST1H2AL, } \\
\text { HIST1H2AM, HIST1H2AJ, HIST1H2AC, HIST2H2AC, HIST1H2BC, HIST1H2BE, HIST1H2BF, HIST1H2BG, } \\
\text { HIST1H2BI, HIST1H2BO, HIST2H2BE, HIST1H3A, HIST1H3B, HIST1H3C, HIST1H3D, HIST1H3E, HIST1H3F, } \\
\text { HIST1H3G, HIST1H3H, HIST1H3I, HIST1H3J, HIST4H4, HIST1H4A, HIST1H4B, HIST1H4C, HIST1H4D, } \\
\text { HIST1H4E, HIST1H4F, HIST1H4H, HIST1H4I, HIST1H4J, HIST1H4K, HIST1H4L, HIST2H4A, HIST2H4B, } \\
\text { HIST1H4G, HIST1H2AH, HIST1H2BK, HIST1H2BJ }\end{array}$ \\
\hline Allograft rejection & 0.046891168 & HLA-DMA, HLA-DMB, HLA-DOA, HLA-DOB, HLA-DPA1, HLA-DQA2, HLA-DRB5, HLA-F, TNF \\
\hline
\end{tabular}

To further explore the expression level of Wnt signaling pathways and its effect on the downstream signaling, WNT1, $\beta$-catenin, c-myc, and cyclin D1 protein expression was measured by IHC in 46 osteosarcoma samples (Figure 4A-D). WNT1 protein expression was detected predominantly in cytoplasm. $\beta$-catenin protein expression was observed in the membrane and cytoplasm but not in the nucleus. c-myc and cyclin D1 protein expression were detected in the nucleus. No protein expression was detected in negative control samples.

WNT1 protein expression was detected in $69.6 \%(32 / 46)$ of the osteosarcomas (Figure 4A), however no $\beta$-catenin protein expression was observed in the nucleus (Figure 4B). $\beta$-catenin protein expression was detected in the membrane and cytoplasm of $69.6 \%(32 / 46)$ of the osteosarcomas (Figure 4B), negative c-myc protein expression was recorded for $52.2 \%(24 / 46)$ of osteosarcomas and negative cyclin D1 protein expression was recorded for $47.8 \%$ $(22 / 46)$ of osteosarcomas (Figure 4C-D). Compared with previously published reports of c-myc and cyclin D1 expression frequencies in other tumors, such as endometrial carcinoma [22,23], these detection frequencies (47.8\% (22/46) c-myc-positive and 52.2\% (24/46) cyclin D1-positive) are low. Combined with the low levels of mRNA expression for Wnt pathway genes, these data, especially the lack of $\beta$-catenin protein expression in the nucleus, suggest that the Wnt signaling pathway may be inactive. 
Table 3 Genetic deletions of key pathway genes in osteosarcoma

\begin{tabular}{|c|c|c|}
\hline KEEG pathways & $P$ value & Gene name cohort \\
\hline Wnt signaling pathway & 0.01039 & $\begin{array}{l}\text { FRAT1, CSNK1A1L, CTBP2, PRICKLE2, FRAT2, GSK3B, PLCB2, SFRP5, MAP3K7, } \\
\text { TCF7L2, WNT5A, WNT6, WNT8B, FZD5, WNT10A, FZD7, FZD8, BTRC }\end{array}$ \\
\hline Hedgehog signaling pathway & 0.012811 & CSNK1A1L, STK36, GSK3B, SUFU, WNT5A, WNT6, WNT8B, WNT1OA, BTRC \\
\hline Adherens junction & 0.014329 & SORBS1, WASF3, FER, FYN, PVRL3, MLLT4, PARD3, MAP3K7, TCF7L2, VCL, WASF1 \\
\hline Metabolism of xenobiotics by cytochrome P450 & 0.006437 & $\begin{array}{l}\text { AKR1C4, GSTO2, CYP2C19, CYP2C8, CYP2C9, CYP2C18, AKR1C1, AKR1C2, } \\
\text { UGT1A8, AKR1C3, GSTO1 }\end{array}$ \\
\hline PPAR signaling pathway & 0.039963 & SORBS1, CYP27A1, FABP7, ACSL3, ACADL, ME1, ACSL5, SCD, ACOX2 \\
\hline Non-homologous end-joining & 0.001607 & DNTT, POLL, DCLRETC, XRCC5, NHEJ1 \\
\hline Phosphatidylinositol signaling system & 0.040426 & DGKH, INPP5D, PLCE1, CALML5, PIK3R1, GNG7, PIP4K2A, PLCB2, PTEN, PLCD4, DGKD \\
\hline ECM-receptor interaction & 0.032505 & COL6A3, FNDC3A, SV2C, FN1, ITGA1, ITGB1, LAMA4, THBS1, THBS2, ITGA8, CD47 \\
\hline T cell receptor signaling pathway & 0.020903 & RASGRP1, CHUK, MAP3K8, CTLA4, FYN, ICOS, NFKB2, PIK3R1, PRKCQ, PAK6, CBLB, CD28 \\
\hline Melanogenesis & 0.007761 & $\begin{array}{l}\text { ADCY5, CREB1, GSK3B, MITF, CALML5, PLCB2, TCF7L2, WNT5A, WNT6, WNT8B, } \\
\text { FZD5, WNT1OA, FZD7, FZD8 }\end{array}$ \\
\hline Basal cell carcinoma & 0.00088 & STK36, GSK3B, SUFU, TCF7L2, WNT5A, WNT6, WNT8B, FZD5, WNT10A, FZD7, FZD8 \\
\hline
\end{tabular}

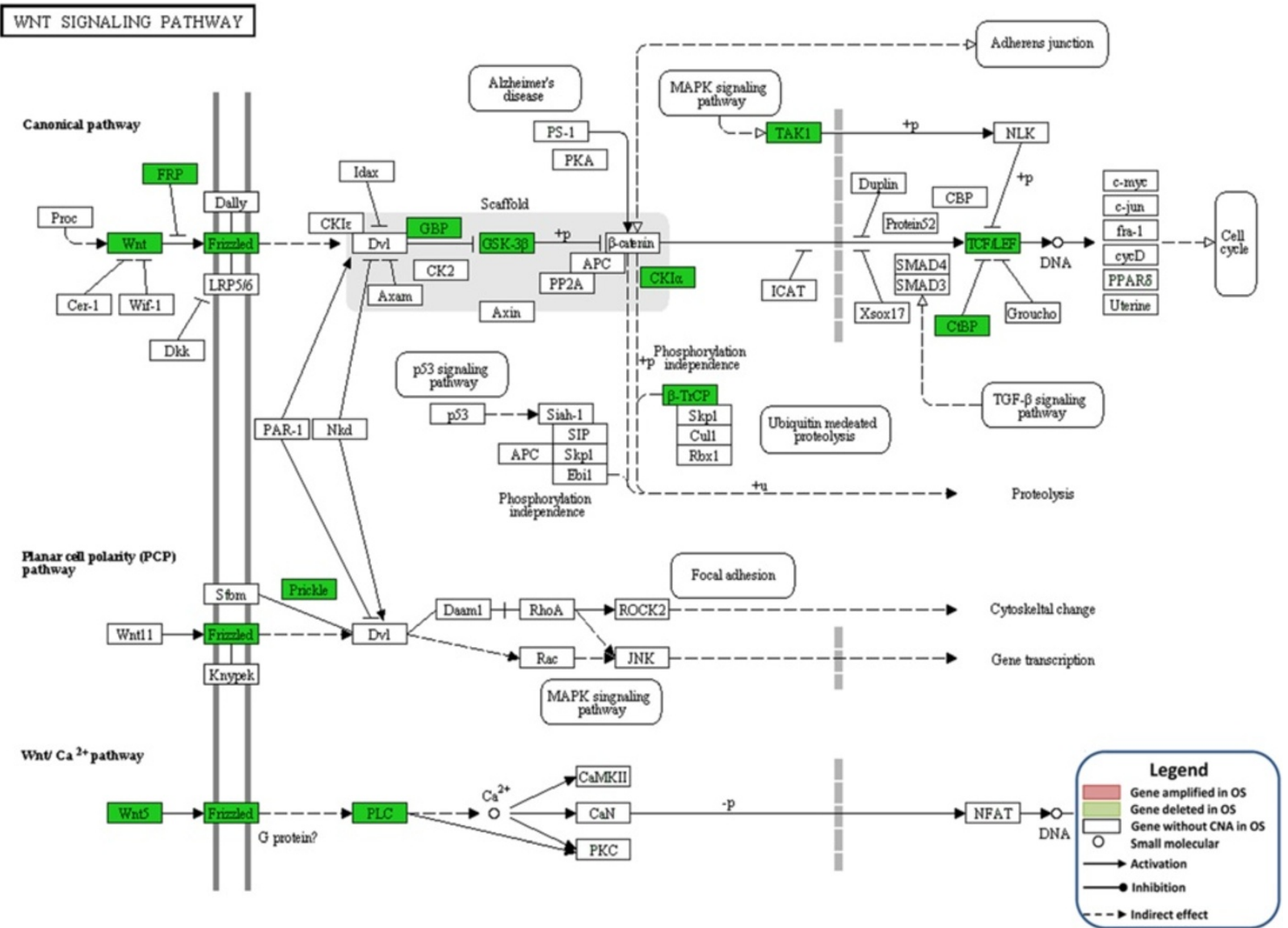

Figure 2 Visualization of the location of altered genes in the Wnt pathway. Pink indicates genes with significantly recurrent amplification, and green denotes genes with significantly recurrent deletion. White indicates genes with no significant aberrations. 


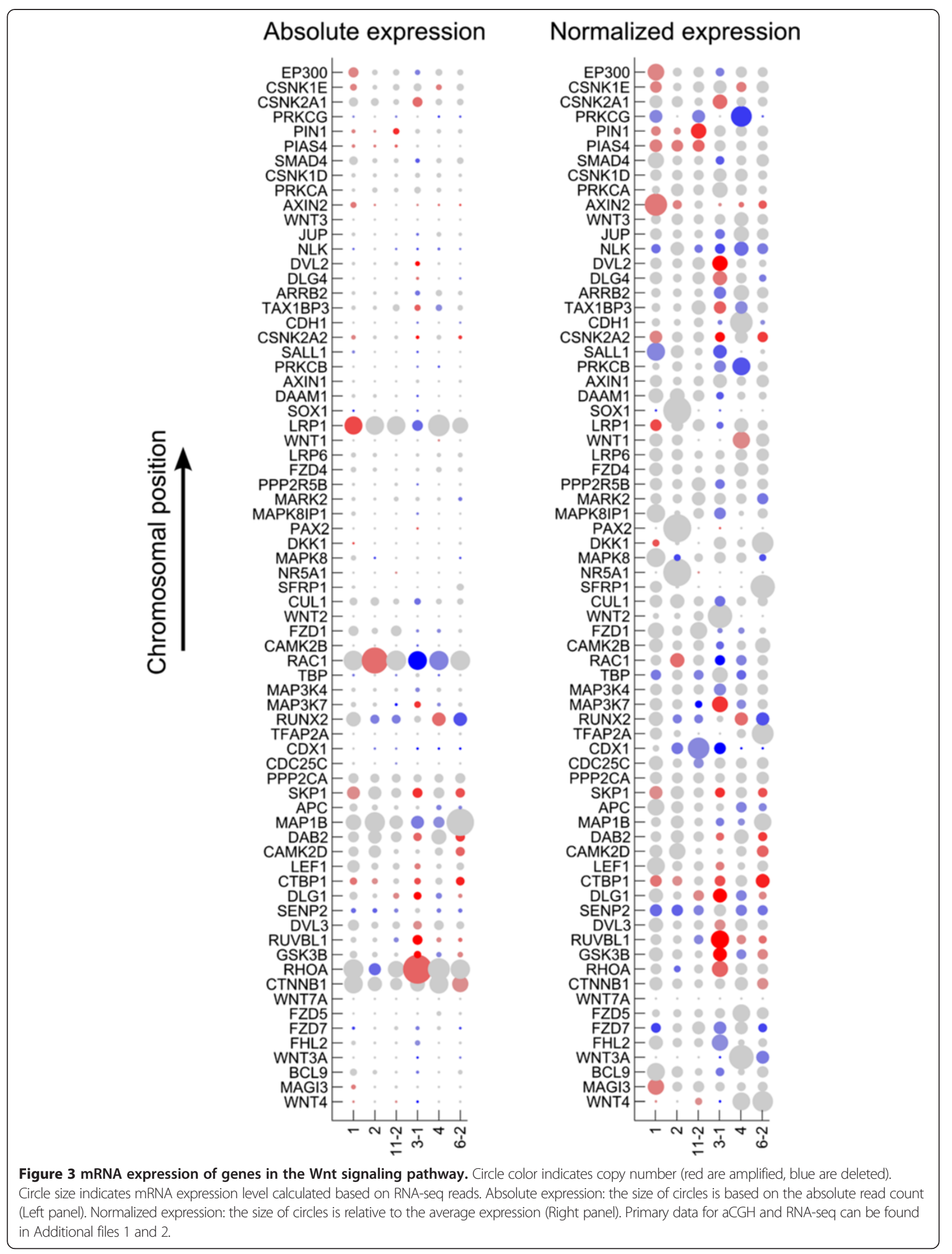




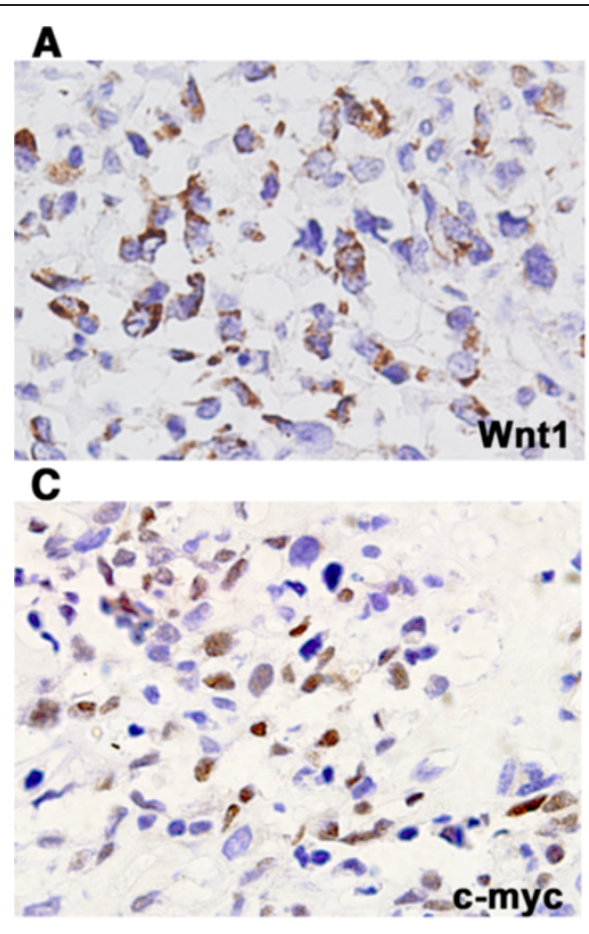

E

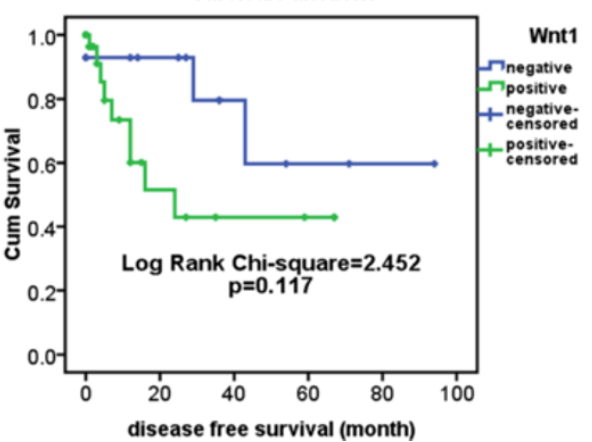

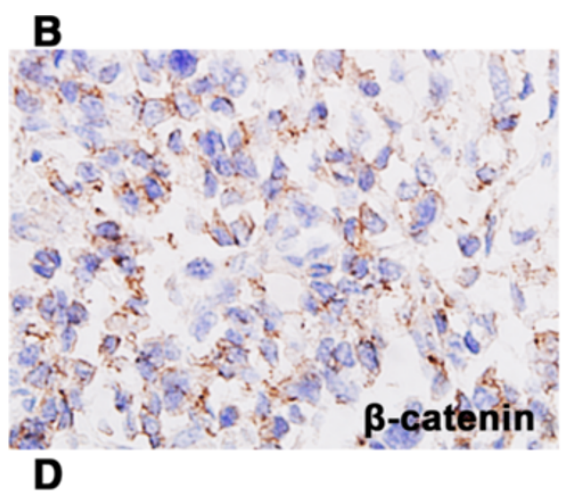

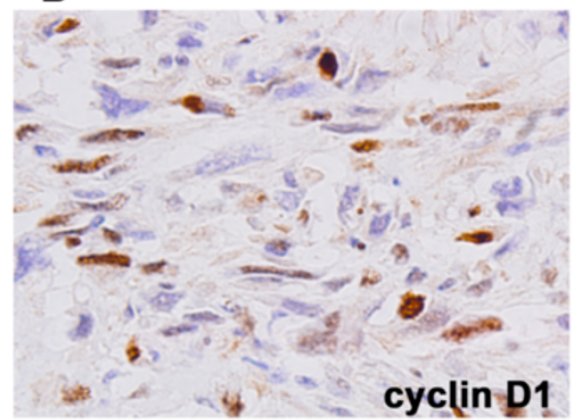

\section{$\mathbf{F}$}

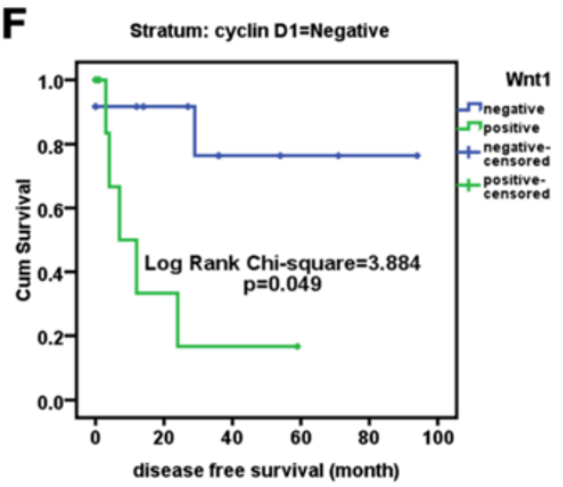

Figure 4 Protein expression of the Wnt signaling pathway and its role in survival. A. WNT1 protein expression (IHC, $10 \times 40)$. B. $\beta-c a t e n i n$ protein expression $(I \mathrm{HC}, 10 \times 40)$. C. c-myc protein expression $(\mathrm{IHC}, 10 \times 40)$. D. Cyclin D1 protein expression $(\mathrm{IHC}, 10 \times 40)$. E. Disease free time of WNT1-negative patients was not significantly longer than WNT1-positive patients. F. K-M survival analysis showed that patients negative for both WNT1 and cyclin D1 expression had significantly longer disease free survival time.

\section{Negative expression of WNT1 and cyclin D1 is associated with longer disease-free survival}

While the expression of WNT1, $\beta$-catenin, c-myc, and cyclin D1 had no correlation with clinicopathological factors, WNT1 expression had significant positive correlation with $\beta$-catenin $\left(X^{2}=15.97, P=0.001\right.$, Pearson's $\mathrm{R}=0.59)$, c-myc $\left(\chi^{2}=5.62, \mathrm{P}=0.018\right.$, Pearson's $\left.\mathrm{R}=0.35\right)$, and cyclin D1 expression $\left(\chi^{2}=11.58, P=0.001\right.$, Pearson's $R=0.50$ ). $\beta$-catenin protein expression had significant positive correlation with c-myc $\left(x^{2}=5.62, \mathrm{p}=0.018\right.$, Pearson's $\mathrm{R}=0.35)$ and cyclin $\mathrm{D} 1\left(\mathrm{x}^{2}=16.36, \mathrm{p}=5.25 \mathrm{E}-5\right.$, Pearson's $R=0.6$ ). Because $\beta$-catenin is a key mediating factor and c-myc/cyclin D1 are key targets regulated by the Wnt signaling pathway, these consistent relationships suggest that the initial signal, mediating factor, and downstream events of the Wnt signaling pathway may all be inactivated in human osteosarcoma.

To detect the effect of the Wnt signaling pathway on survival, the disease-free and total survival of patients were analyzed. Even though single protein expression of $\beta$-catenin, c-myc and cyclin D1 showed no significant effect on disease-free survival, K-M survival analysis showed that WNT1-negative patients had a trend towards longer disease-free survival than WNT1-positive patients, although this was not statistically significant (Log Rank= 2.452, $\mathrm{P}=0.117$ ) (Figure 4E).

Patients negative for all of WNT1, $\beta$-catenin and cyclin D1/c-myc protein expression might be considered to have 
an inactivated Wnt signaling pathway. Therefore, the survival of such patients will reflect the effect of inactivation of the Wnt signaling pathway on survival. Indeed, our data showed that in the patients who were negative for WNT1 protein expression, those who were also negative for cyclin D1 expression had significantly longer disease-free survival than patients with positive cyclin D1 expression (Log Rank $=3.884, \mathrm{P}=0.049$ ) (Figure 4F). However, expression of none of the proteins examined had significant correlation with the overall survival of human osteosarcoma patients.

\section{Discussion}

In this study we describe, for the first time, significant deletion of genes involved in the Wnt signaling pathway, implying genetic inactivation of this important signaling pathway, in human osteosarcoma. Supporting this, transcriptome analysis determined that mRNA expression of genes in the Wnt signaling pathway was reduced. In addition, at the protein level, nuclear $\beta$-catenin expression was not observed and c-myc/cyclin D1 protein were detected at lower frequencies compared with the frequencies observed in other tumors. Our results are in agreement with published data from Cai and colleagues, who reported that the Wnt pathway is inactivated in bone cancers [8]. Furthermore, similar results were reported by Matushansky and Gregory, indicating that inactivation of the Wnt pathway contributes to tumorigenesis in so-called malignant fibrous histiocytoma and melanoma $[17,21,24]$. In contrast to these results, most previous studies have suggested that active Wnt signaling contributes to osteosarcoma development [6,7]. Furthermore, it is reported that the Wnt pathway is transcriptionally active in radiation-induced rat osteosarcomas [25], and that the Wnt/ $\beta$-catenin pathway antagonists, curcumin and PKF118-310, demonstrate anti-tumor activity against human osteosarcoma cells [26]. These seemingly conflicting results suggest that the complexity of this important signaling pathway is still poorly understood in human osteosarcoma [5].

An interesting but unanswered question raised in the present study is: at which time during osteosarcoma progression is the Wnt signaling pathway inactivated. As inactivation of the Wnt signaling pathway is associated with longer disease-free survival, it suggests that this inactivation may occur at an early time point in osteosarcoma progression. However, more investigations in vivo and in vitro are required before this question can be answered.

\section{Conclusions}

Based on evidence at the genomic, our data suggest that the Wnt signaling pathway may be genetically inactivated in osteosarcoma. These results remind us of the complexity of this important signaling pathway.

\section{Additional files} Additional file 1: Gene differential expression of osteosarcoma
samples.

Additional file 2: The significant deletion of Wnt signal pathway genes.

\section{Competing interests}

The authors declare that they have no competing interests.

\section{Authors' contributions}

$X D, J Y$, and DY carried out the molecular genetic studies, participated in the sequence alignment and drafted the manuscript. XD, DY, and WT carried out the immunohistochemistry. JY, DY, and ZZ participated in the design of the study. JY and ZZ conceived of the study, and participated in its design and coordination and helped to draft the manuscript. All authors read and approved the final manuscript.

\section{Acknowledgments}

This work was partly supported by the National Nature Science Foundation of China (81372872 to JY and 81320108022 to KC), the funds from the

University Cancer Foundation via the Sister Institution Network Fund (SINF) at the Tianjin Medical University Cancer Institute \& Hospital (TMUCIH), Fudan University Shanghai Cancer Center (FUSCC), and University of Texas MD Anderson Cancer Center (UT MDACC), program for Changjiang Scholars and Innovative Research Team in University (PCSIRT) in China (IRT1076), and National Key Scientific and Technological Project (2011ZX09307-001-04) (K. Chen).

The genomic studies were supported by Dr. Wei Zhang

(wzhang@mdanderson.org) and the Cancer Genomics Core Laboratory. We would like to thank Limei Hu and David Cogdell for performing the aCGH experiments.

\section{Author details}

${ }^{1}$ Department of Diagnostics, Tianjin Medical University, Tianjin 300060, China. ${ }^{2}$ Department of Bone and Soft Tissue Tumors, Tianjin Medical University Cancer Institute \& Hospital, National Clinical Research Center for Cancer, Tianjin 30060, China. ${ }^{3}$ Department of Pathology, The University of Texas MD Anderson Cancer Center, Houston, TX 77030, USA. ${ }^{4}$ Department of Medical Microbiology, Tianjin Medical University, Tianjin 300060, China.

Received: 27 December 2013 Accepted: 5 June 2014

Published: 18 June 2014

\section{References}

1. Picci $P$, Mercuri M, Ferrari S, Alberghini M, Briccoli A, Ferrari C, Pignotti E, Bacci G: Survival in high-grade osteosarcoma: improvement over 21 years at a single institution. Ann Oncol 2010, 21(6):1366-1373.

2. Yang J, Yang D, Sun Y, Sun B, Wang G, Trent JC, Araujo DM, Chen K, Zhang $W$ : Genetic amplification of the vascular endothelial growth factor (VEGF) pathway genes, including VEGFA, in human osteosarcoma. Cancer 2011, 117(21):4925-4938.

3. Yang J, Cogdell D, Yang D, Hu L, Li H, Zheng H, Du X, Pang Y, Trent J, Chen $K$, Zhang W: Deletion of the WWOX gene and frequent loss of its protein expression in human osteosarcoma. Cancer Lett 2010, 291(1):31-38.

4. Bjornsti MA, Houghton PJ: The TOR pathway: a target for cancer therapy. Nat Rev Cancer 2004, 4(5):335-348.

5. Thomas DM: Wnts, bone and cancer. J Pathol 2010, 220(1):1-4.

6. Iwaya K, Ogawa H, Kuroda M, Izumi M, Ishida T, Mukai K: Cytoplasmic and/ or nuclear staining of beta-catenin is associated with lung metastasis. Clin Exp Metastasis 2003, 20(6):525-529.

7. Kansara M, Tsang M, Kodjabachian L, Sims NA, Trivett MK, Ehrich M, Dobrovic A, Slavin J, Choong PF, Simmons PJ, Dawid IB, Thomas DM: Wnt inhibitory factor 1 is epigenetically silenced in human osteosarcoma, and targeted disruption accelerates osteosarcomagenesis in mice. J Clin Invest 2009, 119(4):837-851.

8. Cai Y, Mohseny AB, Karperien M, Hogendoorn PC, Zhou G, Cleton-Jansen AM: Inactive Wnt/beta-catenin pathway in conventional high-grade osteosarcoma. J Pathol 2010, 220(1):24-33. 
9. Meyers PA, Heller G, Healey J, Huvos A, Lane J, Marcove R, Applewhite A, Vlamis V, Rosen G: Chemotherapy for nonmetastatic osteogenic sarcoma: the Memorial Sloan-Kettering experience. J Clin Oncol 1992, 10(1):5-15.

10. Boussen H, Mezzi F, Gamoudi A, Daldoul O, Ben Hamida H, Mezlini A, Khalfallah S, Karray S, Ben Romdhane K, Ben Ghachem M, Ben Abdallah M, Douik M, Saadi A, Ben Ayed F, Ben Hassine H: [Primary chemotherapy with the Rosen T10 protocol before conservative surgery in limb primitive osteosarcomas: results about 56 cases]. Bull Cancer 2000, 87(2):183-188.

11. Squire JA, Pei J, Marrano P, Beheshti B, Bayani J, Lim G, Moldovan L, Zielenska M: High-resolution mapping of amplifications and deletions in pediatric osteosarcoma by use of CGH analysis of CDNA microarrays. Genes Chromosomes Cancer 2003, 38(3):215-225.

12. Olshen $A B$, Venkatraman ES, Lucito R, Wigler M: Circular binary segmentation for the analysis of array-based DNA copy number data. Biostatistics 2004, 5(4):557-572.

13. Van Wieringen WN, Van De Wiel MA, Ylstra B: Weighted clustering of called array CGH data. Biostatistics 2008, 9(3):484-500.

14. Kresse SH, Ohnstad HO, Paulsen EB, Bjerkehagen B, Szuhai K, Serra M, Schaefer KL, Myklebost O, Meza-Zepeda LA: LSAMP, a novel candidate tumor suppressor gene in human osteosarcomas, identified by array comparative genomic hybridization. Genes Chromosomes Cancer 2009, 48(8):679-693.

15. Kim D, Pertea G, Trapnell C, Pimentel H, Kelley R, Salzberg SL: TopHat2: accurate alignment of transcriptomes in the presence of insertions, deletions and gene fusions. Genome Biol 2013, 14(4):R36.

16. Mortazavi A, Williams BA, McCue K, Schaeffer L, Wold B: Mapping and quantifying mammalian transcriptomes by RNA-Seq. Nat Methods 2008, 5(7):621-628.

17. Chien AJ, Moore EC, Lonsdorf AS, Kulikauskas RM, Rothberg BG, Berger AJ, Major MB, Hwang ST, Rimm DL, Moon RT: Activated Wnt/beta-catenin signaling in melanoma is associated with decreased proliferation in patient tumors and a murine melanoma model. Proc Natl Acad Sci U S A 2009, 106(4):1193-1198.

18. De Blasio A, Messina C, Santulli A, Mangano V, Di Leonardo E, D'Anneo A, Tesoriere G, Vento R: Differentiative pathway activated by 3-aminobenzamide, an inhibitor of PARP, in human osteosarcoma MG-63 cells. FEBS Lett 2005, 579(3):615-620

19. Gazitt Y, Kolaparthi V, Moncada K, Thomas C, Freeman J: Targeted therapy of human osteosarcoma with 17AAG or rapamycin: characterization of induced apoptosis and inhibition of mTOR and Akt/MAPK/Wnt pathways. Int J Oncol 2009, 34(2):551-561.

20. Takayama S, Rogatsky I, Schwarcz LE, Darimont BD: The glucocorticoid receptor represses cyclin D1 by targeting the Tcf-beta-catenin complex. J Biol Chem 2006, 281(26):17856-17863.

21. Matushansky I, Hernando E, Socci ND, Mills JE, Matos TA, Edgar MA, Singer S, Maki RG, Cordon-Cardo C: Derivation of sarcomas from mesenchymal stem cells via inactivation of the Wnt pathway. J Clin Invest 2007, 117(11):3248-3257.

22. Gu Y, Pan Y, Meng B, Guan B, Fu K, Sun B, Zheng F: High levels of bcl-2 protein expression do not correlate with genetic abnormalities but predict worse prognosis in patients with lymphoblastic lymphoma. Tumour Biol 2013, 34(3):1441-1450.

23. Liang S, Mu K, Wang Y, Zhou Z, Zhang J, Sheng Y, Zhang T: CyclinD1, a prominent prognostic marker for endometrial diseases. Diagn Pathol 2013, 8:138.

24. Gregory CA, Singh H, Perry AS, Prockop DJ: The Wnt signaling inhibitor dickkopf-1 is required for reentry into the cell cycle of human adult stem cells from bone marrow. J Biol Chem 2003, 278(30):28067-28078.

25. Daino K, Ugolin N, Altmeyer-Morel S, Guilly MN, Chevillard S: Gene expression profiling of alpha-radiation-induced rat osteosarcomas: identification of dysregulated genes involved in radiation-induced tumorigenesis of bone. Int J Cancer 2009, 125(3):612-620.

26. Leow PCTQ, Ong ZY, Yang Z, Ee PL: Antitumor activity of natural compounds, curcumin and p KF118-310, as Wnt/ $\beta$-catenin antagonists against human osteosarcoma cells. Invest New Drugs 2010, 28(6):766-782

\section{Submit your next manuscript to BioMed Central and take full advantage of:}

- Convenient online submission

- Thorough peer review

- No space constraints or color figure charges

- Immediate publication on acceptance

- Inclusion in PubMed, CAS, Scopus and Google Scholar

- Research which is freely available for redistribution 